Bulletin of Mathematical Biology (1999) 61, 483-505

Article No. bulm.1998.0093

Available online at http://www.idealibrary.com on IDE $\mathbf{A}^{(\mathbb{1}}$

\title{
A Two-dimensional Numerical Study of Spatial Pattern Formation in Interacting Turing Systems
}

\author{
R. A. BARRIO, C. VAREA, J. L. ARAGÓN \\ Instituto de Física, UNAM, Apartado Postal 20-364, \\ 01000 México, D.F., Mexico \\ P. K. MAINI \\ Centre for Mathematical Biology, \\ Mathematical Institute, University of Oxford, \\ 24-29 St. Giles, Oxford OX1 3LB, U.K.
}

\begin{abstract}
For many years Turing systems have been proposed to account for spatial and spatiotemporal pattern formation in chemistry and biology. We extend the study of Turing systems to investigate the rôle of boundary conditions, domain shape, non-linearities, and coupling of such systems. We show that such modifications lead to a wide variety of patterns that bear a striking resemblance to pigmentation patterns in fish, particularly those involving stripes, spots and transitions between them. Using the Turing system as a metaphor for activator-inhibitor models we conclude that such a mechanism, with the aforementioned modifications, may play a rôle in fish patterning.
\end{abstract}

(C) 1999 Society for Mathematical Biology

\section{INTRODUCTION}

One of the crucial areas of research in developmental biology concerns the formation of spatial pattern in the early embryo. From a single cell with limited spatial structure, emerges the vast range of patterns that we see in nature, such as skeletal patterns, hair, teeth, feathers and coat markings. Although genes play a vital rôle in embryological development, a study of genetics alone is not sufficient to understand the generation and subsequent interpretation of the complex spatiotemporal signaling cues that occur in early development. These arise as the result of the complex interaction of many physical and chemical processes. The outcome of such non-linear interactions is best understood through mathematical modeling, and the rôle of modeling in many areas of developmental biology is to help understand how biologically plausible processes interact.

One of the most widely studied models for spatial pattern formation is that proposed by Turing (1952). He showed that a system of two reacting and diffusing chemicals could give rise to spatial pattern in chemical concentrations from initial near-homogeneity. This phenomenon, termed diffusion-driven instability, has now been shown to occur in chemistry. Experimental results illustrate the formation of $0092-8240 / 99 / 030483+23 \quad \$ 30.00 / 0$ (C) 1999 Society for Mathematical Biology 
striped and spotted patterns, as well as more complicated patterns. Many of these patterns can be exhibited by Turing models and there is now a vast amount of theoretical and experimental literature in this area [see Maini et al. (1997), for a review]. Reaction-diffusion theory has been used in biological pattern formation by assuming that the non-uniform chemical concentrations arising from diffusion-driven instability act as a pre-pattern to which cells respond and differentiate accordingly.

An alternative mechanism for spatial patterning is the mechanochemical approach [see Murray (1993), for a comprehensive review]. This assumes that pattern arises due to the physical interaction of cells with their external environment, leading to cell aggregations which then differentiate accordingly.

Both these models have been extensively studied and it has been shown that they can exhibit many patterns that are observed in nature. In particular, the effects of domain size and non-linearities on the selection and stability of solutions has been analysed. Specifically, it has been shown that the absence of quadratic nonlinearities in the reaction kinetics favors stripes, but the presence of quadratic terms favors spots (Ermentrout, 1991; Nagorcka and Mooney, 1992).

Many developmental phenomena, for example, the formation of skin organs, arise as the result of tissue interaction. In such cases, one must consider the coupling of pattern generator models. For example, Nagorcka et al. (1987) developed an interaction model that coupled a mechanochemical system with a reaction-diffusion system. This model was analysed by Shaw and Murray (1990) and shown to exhibit, in one dimension, complex spatial patterns of superimposed different wavelengths. Both these papers presented a limited, preliminary study of patterns on two-dimensional rectangular domains. Cruywagen et al. $(1992 ; 1997)$ and Murray et al. (1994) have extensively analysed the coupled mechanochemical model for tissue interaction proposed by Cruywagen and Murray (1992). They have shown that each sub-model is unable to generate patterns, but the coupling of the models leads to the development of patterns. They showed that in two dimensions, complex propagating patterns could arise, the form of which depended crucially on initial conditions.

More recently, Painter et al. (1999) have considered the weak coupling of a chemotactic cell aggregation model with a reaction-diffusion system and shown that the resultant model can exhibit complex spatial patterns, such as stripes of different widths, that are observed biologically but cannot be generated by a single reaction-diffusion system. Höfer and Maini (1997) have shown how the coupling of a chemotactic mechanism with an excitable reaction-diffusion system can lead to cell aggregation via cell streaming, as observed in the slime mould Dictyostelium discoideum.

Much of the detailed investigation of the results of coupling pattern generators has been carried out in one dimension, or has been done in two dimensions for simple forms of coupling on rectangular domains with zero flux boundary conditions. In the present paper, we aim to extend this study by considering the results of coupling, in a number of ways, for two reaction-diffusion systems. We chose this particular 
system because it is simpler than the mechanochemical model, yet the patterns generated by both reaction-diffusion and mechanochemical models have thus far been shown to be similar.

In Section 2 we consider first a single, generic, reaction-diffusion model on a two-dimensional domain and verify previous results on the effects of the form of the non-linearity on the selection of pattern. The model kinetics that we consider are not derived from a chemical reaction, rather they incorporate the key elements (linear and non-linear) that have been shown to be crucial in pattern selection in models which do have a firmer biological or chemical basis. The simplicity of our model kinetics makes transparent the effects and interaction of these terms, and our results apply to more realistic models. In Section 3 we study the effects of confining the domains to certain shapes and the possible existence of sources or sinks of chemicals at the boundaries. In Section 4 we consider the coupling of two reaction-diffusion models and investigate the effects of different types of coupling. In Section 5 we show that the complex patterns generated by our models bear a striking similarity to pigmentation patterns observed on a number of fish species.

\section{THE MODEL}

Our main objective is to investigate the patterns formed in confined two-dimensional finite domains by Turing mechanisms. Turing systems have the form

$$
\begin{aligned}
& \frac{\partial U}{\partial t}=D_{u} \nabla^{2} U+f(U, V) \\
& \frac{\partial V}{\partial t}=D_{v} \nabla^{2} V+g(U, V) .
\end{aligned}
$$

These equations describe the evolution of the concentrations, $U(\bar{x}, t), V(\bar{x}, t)$ at spatial position $\bar{x}$ and time $t$, of two chemicals due to diffusion, with constant diffusion coefficients $D_{u}, D_{v}$, respectively, and reaction, modeled by the (typically non-linear) functions, $f$ and $g$. The review by Maini et al. (1997) considers the commonly used reaction kinetics and presents their motivation.

The above equations are solved on some bounded domain $\Omega \subset R^{n}$ with boundary conditions which may be of Neumann, Dirichlet, Robin or periodic type. The concentrations of the chemicals are specified at $t=0, \forall \bar{x} \in \Omega$.

A spatially-uniform steady state of the above system is the state $\left(U_{c}, V_{c}\right)$ such that $f\left(U_{c}, V_{c}\right)=g\left(U_{c}, V_{c}\right)=0$ and such that $U=U_{c}, V=V_{c}$ satisfies the boundary conditions. Turing showed that under certain conditions on the parameter values, such a steady state could be linearly stable in the absence of diffusion but unstable in the presence of diffusion - this is the now well-known phenomenon of diffusiondriven instability. For example, on a rectangular two-dimensional domain with zero flux boundary conditions, the following conditions must be satisfied for diffusiondriven instability: 


$$
\begin{aligned}
& \text { (I) } \frac{\partial f}{\partial U}+\frac{\partial g}{\partial V}<0 \\
& \text { (II) } \frac{\partial f}{\partial U} \frac{\partial g}{\partial V}-\frac{\partial f}{\partial V} \frac{\partial g}{\partial U}>0 ; \\
& \text { (III) } D_{U} \frac{\partial g}{\partial V}+D_{V} \frac{\partial f}{\partial U}>0 ; \\
& \text { (IV) } D_{U} \frac{\partial g}{\partial V}+D_{V} \frac{\partial f}{\partial U}>2 \sqrt{D_{U} D_{V}\left(\frac{\partial f}{\partial U} \frac{\partial g}{\partial V}-\frac{\partial f}{\partial V} \frac{\partial g}{\partial U}\right)}
\end{aligned}
$$

where the partial derivatives of $f$ and $g$ are evaluated at $\left(U_{c}, V_{c}\right)$. As a further condition, the uniform steady state can only go unstable to modes that have positive growth rate and satisfy the boundary conditions - the so-called admissible modes.

On two-dimensional domains, there can be a number of different admissible modes with the same wavenumber. In such a degenerate case, the question naturally arises of which mode will dominate in the full non-linear system. The papers by Ermentrout (1991), and Nagorcka and Mooney (1992) show that the form of the non-linearity [whether it is quadratic or cubic when expanded about $\left(U_{c}, V_{c}\right)$ ] is the crucial factor in pattern selection.

As our starting point, we propose a simple set of reaction-diffusion equations which encompasses all the above features. The basic equations are obtained from equation (1) in the following way. We first observe that, in general, there is a stationary uniform solution $\left(U_{c}, V_{c}\right)$, given by the zeros of $f$ and $g$. We then expand the functions around this point in a Taylor series, neglecting terms of order higher than cubic. The specific form we consider is:

$$
\begin{aligned}
& \frac{\partial u}{\partial t}=D \delta \nabla^{2} u+\alpha u\left(1-r_{1} v^{2}\right)+v\left(1-r_{2} u\right) \\
& \frac{\partial v}{\partial t}=\delta \nabla^{2} v+\beta v\left(1+\frac{\alpha r_{1}}{\beta} u v\right)+u\left(\gamma+r_{2} v\right),
\end{aligned}
$$

where $u=U-U_{c}$ and $v=V-V_{c}$, so the uniform stationary solution of equation (2) is the point $(0,0)$. The special arrangement of the coefficients is dictated by the conservation relation between chemicals. The quantity $\delta$ is the ratio between diffusion coefficients of the two chemicals, and must not be equal to unity in order to satisfy conditions I-IV. There are two interaction parameters, $r_{1}$ and $r_{2}$, corresponding to a cubic and a quadratic term, respectively. It is widely known that a cubic interaction favors stripes and that a quadratic one produces spot patterns. The wavelengths present in the patterns depend on the values of the coefficients, which select the modes to which the uniform steady state is linearly unstable. 
2.1. Linear analysis. In the absence of diffusion, equation (2) shows another stationary uniform solution at

$$
v=\frac{-(\alpha+\gamma)}{1+\beta} u
$$

To keep the investigation as simple as possible, we enforce $(0,0)$ to be the only spatially-uniform steady state by setting $\alpha=-\gamma$. In the absence of diffusion, standard linear analysis predicts exponentially growing solutions of the form $(u, v)=\left(u_{0} \exp (\lambda t), v_{0} \exp (\lambda t)\right)$, with

$$
\lambda=\frac{1}{2}\left[(\alpha+\beta) \pm \sqrt{(\alpha+\beta)^{2}-4(\alpha \beta-\gamma)}\right] .
$$

We require the uniform solution to be linearly stable, therefore $\operatorname{Re}(\lambda)$ must be less than zero. This will hold for either $\alpha \geq 0$ and $\beta \leq-\alpha$, or for $\alpha \leq 0$ and $\beta \leq-1$ (cf. conditions I and II above).

In the presence of diffusion, the spatial variation of the functions $u$ and $v$ is of the form $\exp (i \bar{k} \cdot \bar{x})$, and the dispersion relation of the linearized equations is given by the solutions of

$$
\lambda^{2}-B \lambda+C=0,
$$

where $B=k^{2} \delta(1+D)$ and $C=\left(\alpha-\delta D k^{2}\right)\left(\beta-\delta k^{2}\right)+\alpha, k^{2}=\bar{k} \cdot \bar{k}$. The solutions of $C=0$ are the bounds, in $k$ space, of a region with positive $\lambda$. These bounds need to be real positive, which implies the condition $\alpha-2 \sqrt{\alpha D}>\beta D$. On a confined spatial domain, $k$ is discrete (corresponding to admissible modes), and the quantity $\delta=2$ gives the scale of the size of the domain. Therefore, we now have as free parameters, $D, \alpha$ and $\beta$ and the values of these determine which modes evolve. We are specifically interested in studying the formation of stripes and spots, so we choose these parameters in such a way that very few admissible modes have positive growth rates in a grid of size $L \times L$, and that at least one of the form $\bar{k}=2 \pi(n / L, 0)$ (corresponding to stripes) and another of the form $\bar{k}=2 \pi(m / L, m / L)$ (corresponding to spots) are present. Due to the complicated algebraic form of $B$ and $C$ it is very difficult to choose parameters that isolate specific modes of the aforementioned form, so in the following, when we talk about a given $\bar{k}$, we will be referring to the monomode that corresponds to the maximum value of $\lambda$.

In Fig. 1 we show the real part of two pairs of eigenvalues corresponding to two different sets of parameters as a function of the wave vector. The parameters were chosen to enhance modes of the form $\bar{k}=2 \pi(4 / 60,0)(k=0.42)$ and $\bar{k}=2 \pi(8 / 60,0)(k=0.84)$, respectively.

2.2. Numerical calculations. We first consider equation (2) on a square domain and solve it on a grid with $100 \times 100$ sites by a simple Euler method with a time 


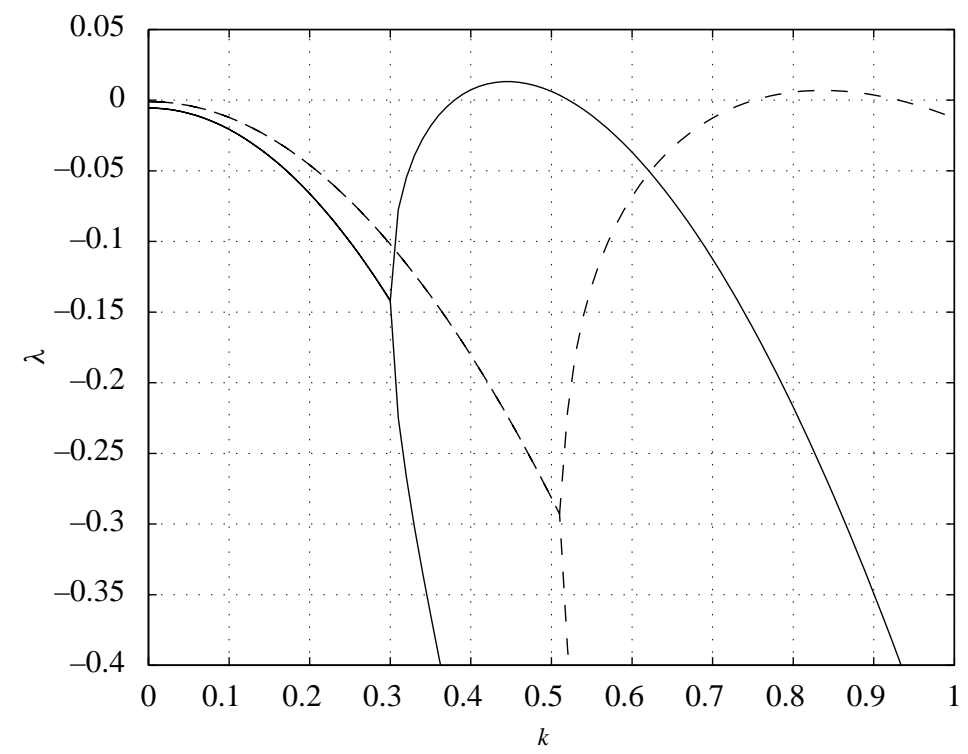

Figure 1. Eigenvalues of the linearized characteristic matrix of equation (2). The admissible modes in a finite lattice have discrete wave numbers $k=2 \pi \sqrt{\left(n / L_{x}\right)^{2}+\left(m / L_{y}\right)^{2}}$, where $L_{x}$ and $L_{y}$ are the number of pixels in the $x$ and $y$ directions, respectively. The parameter values $D=0.516, \alpha=0.899$, and $\beta=-0.91$, were used to enhance the mode $k=$ $2 \pi(4 / 60)=0.42$ (continuous line) in a grid of $30 \times 30$ with $\delta=2$, while values $D=0.122$, $\alpha=0.398$, and $\beta=-0.4$ enhance the mode $k=2 \pi(8 / 60)=0.84$ (dashed line).

step of $\Delta t=0.05$, and by discretizing the Laplacian in the grid with lattice sites denoted by $(i, j)$. The form is

$$
\left.\nabla^{2} u\right|_{(i, j)}=\frac{1}{b^{2}}\left(\begin{array}{l}
{\left[a_{r}(i, j) u(i+1, j)-u(i, j)\right]+} \\
{\left[a_{l}(i, j) u(i-1, j)-u(i, j)\right]+} \\
{\left[a_{u}(i, j) u(i, j+1)-u(i, j)\right]+} \\
{\left[a_{d}(i, j) u(i, j-1)-u(i, j)\right]}
\end{array}\right),
$$

where $b$ is the lattice constant and the matrix elements of $a_{r}, a_{l}, a_{u}$ and $a_{d}$ are unity except at the boundary where they are set equal to zero when the lattice site has no $\operatorname{right}(r), \operatorname{left}(l), \operatorname{up}(u)$ or down $(d)$ neighbor, respectively. This ensures zero flux of reactants in or out of the boundary, and it is equivalent to setting $\hat{n} \cdot \nabla u=0$ at the boundary with normal vector $\hat{n}$.

The stable patterns obtained can either be spots or stripes, depending on the values of $r_{1}$ and $r_{2}$. It is seen that the quadratic term $r_{2}$ favors spots while the cubic term $r_{1}$ produces stripes. In Fig. 2 we show some examples of these simple cases.

Our extensive numerical simulations suggest that, in general, spots are more robust and that the amplitude differences are larger than those for stripes, that is, spots are more pronounced. The stripes are only formed for very small values of $r_{2}$, and can be oriented in any direction, depending on the initial conditions. In these 

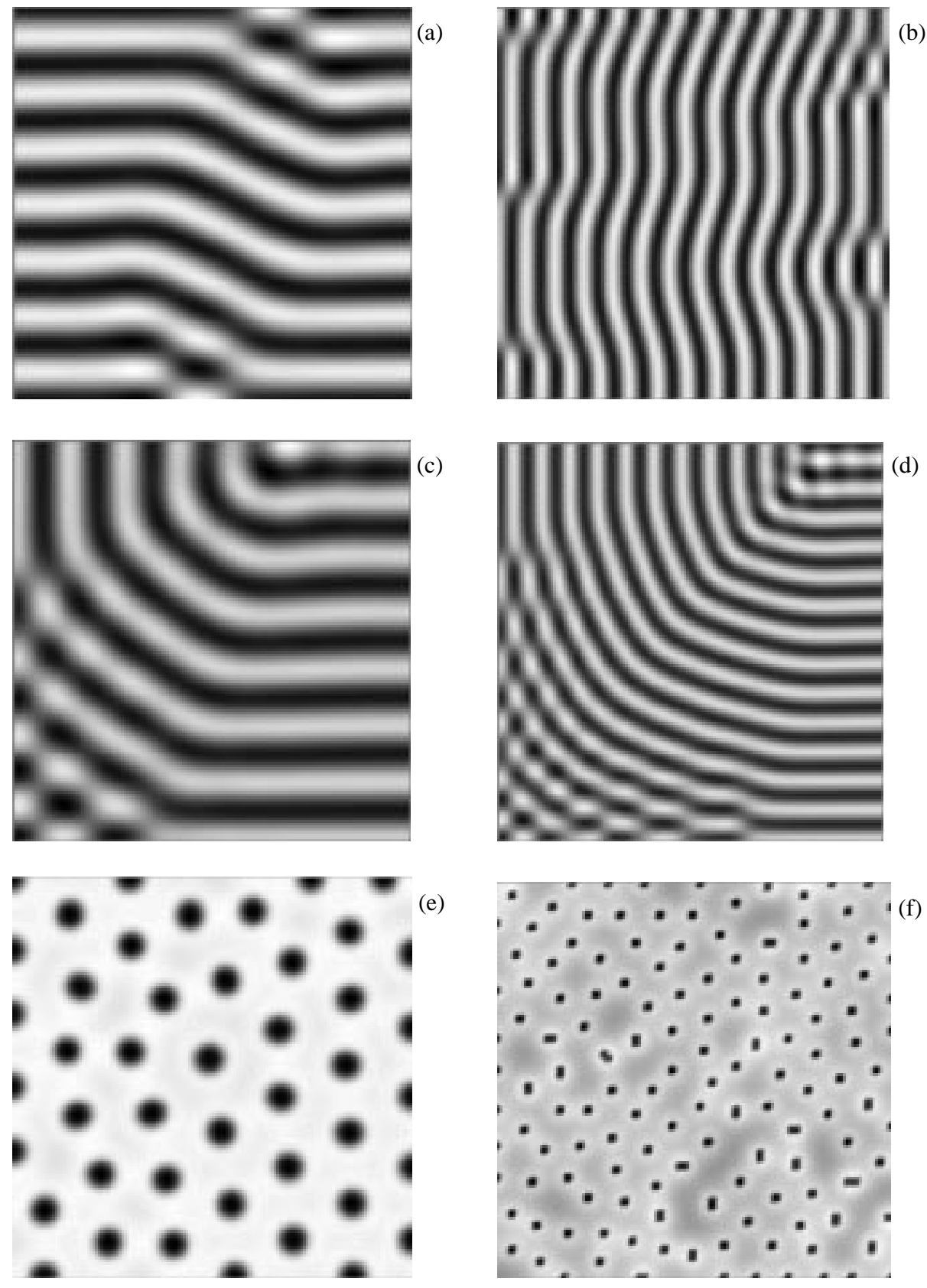

Figure 2. Patterns obtained with equation (2): (a) parameters $r_{1}=3.5$ and $r_{2}=0$, other parameters chosen so that mode $k=0.42$ is selected in the linear regime; (b) same as (a) but with parameters chosen to enhance mode $k=0.84$; (c) and (d) are the same as (a) and (b), respectively, except that the starting random seed is different; (e) and (f) correspond to the same modes as before but the non-linear parameters are $r_{1}=0.02$ and $r_{2}=0.2$. 


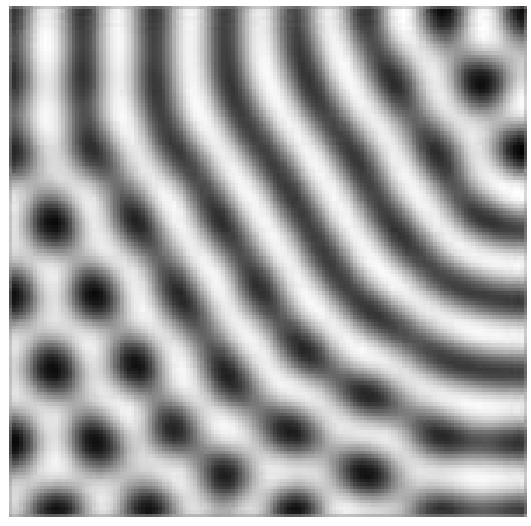

160000 Iterations

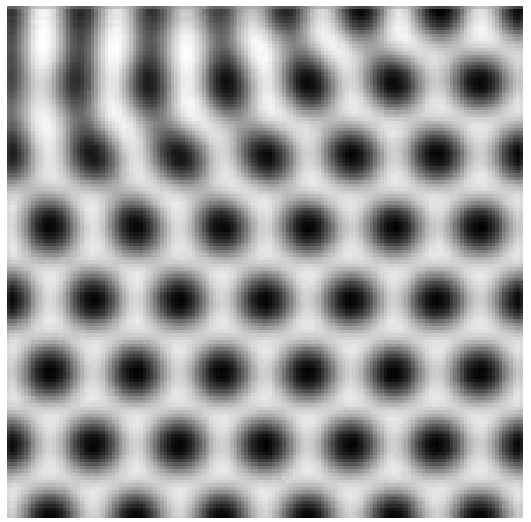

640000 Iterations

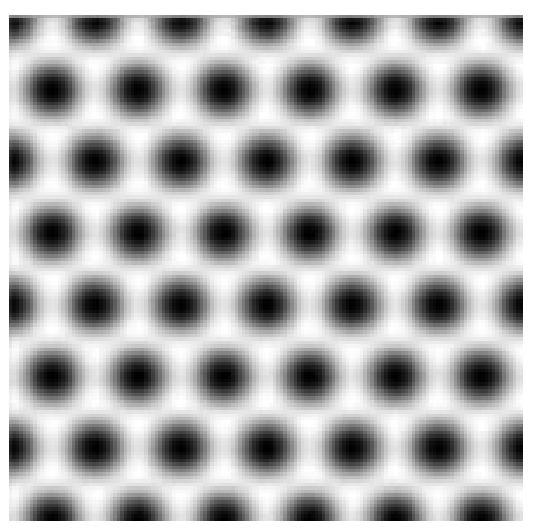

960000 Iterations

Figure 3. Patterns obtained with equation (2) for $r_{1}=3.5$ and $r_{2}=0.2$. The other parameters are as in the first column of Fig. 2. The figure shows snaps of the patterns taken at increasing times. Observe that the transient patterns present stripes and spots, but the converged patterns only present spots arranged in a perfectly hexagonal lattice.

calculations, we started with random values having a flat distribution and different seeds.

In Fig. 3 we show a competition between stripes and spots when both non-linear parameters are different from zero. The pattern takes a long time to settle down, starting with stripes and spots and ending with a perfect hexagonal lattice of spots only.

\section{BOUNDARY AND SHAPE EFFECTS}

It is known that patterns produced by simple Turing systems can be sensitive to domain shape (Bunow et al., 1981) and, therefore, it is important to investigate the robustness of the above patterns to changes in geometry. Our aim is to investigate 


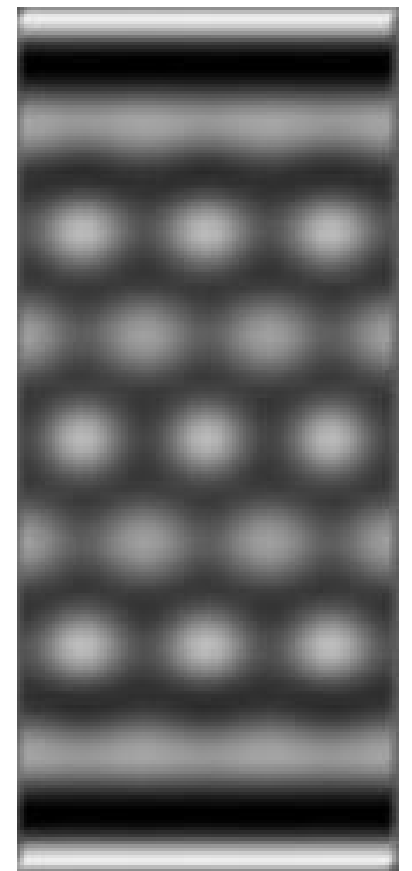

(a)

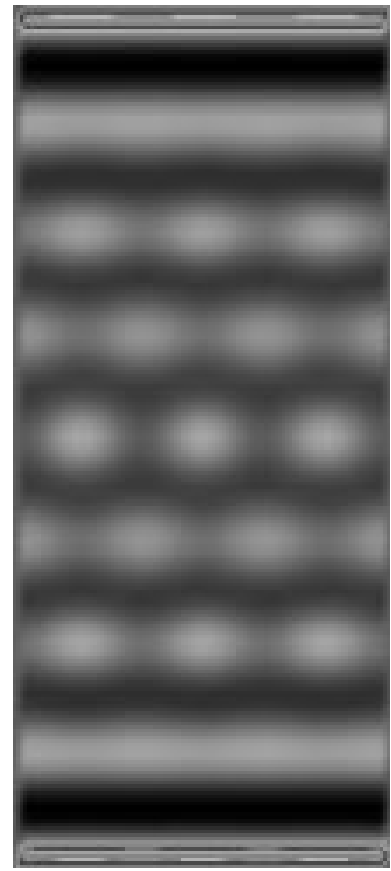

(b)

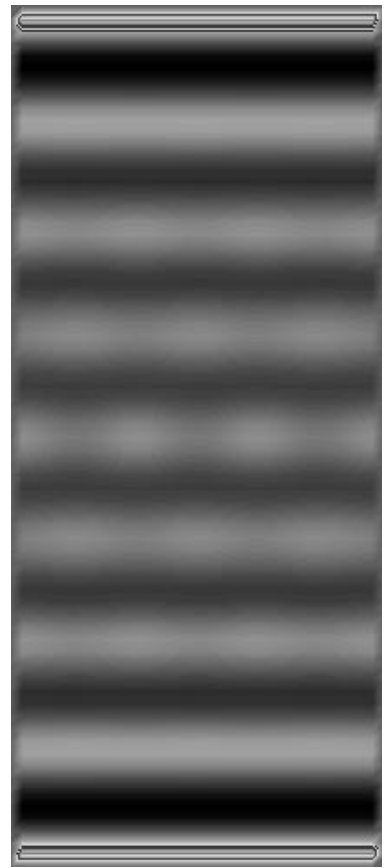

(c)

Figure 4. Patterns obtained with parameter values $D=0.182, \alpha=0.5, \beta=-0.578$ and $r_{1}=0.5$, with a source $h=0.005$ of $u$ at the top and the bottom boundaries. The parameter $r_{2}$ has values: (a) 0.47 ; (b) 0.35 ; and (c) 0.29 . Observe the increasing tendency to form stripes as $r_{2}$ decreases.

the above system as a possible mechanism for pigmentation patterning in fish, so we first investigate how the pattern changes due to changes in the shape of the domain. Specifically, we consider a simple approximation to a fish shape, consisting of three parabolic edges and one straight edge. We find that there is no detectable difference with the patterns already shown.

Next, we consider the rôle of boundary conditions, as it is known that these can have a pronounced effect on the pattern exhibited by simple Turing systems (Dillon et al., 1994; Varea et al., 1997). In this case, we find that the existence of a source of chemical at the borders dramatically changes the patterns.

As a first example, in Fig. 4 we show the effect of introducing a constant source term $h$ in the $u$ in equation (2), at the upper and lower boundaries of a rectangular domain. The three patterns correspond to increasing values of $r_{2}$, starting with a value that gives a spot pattern in the absence of the source terms. Observe that the source produces a pattern with stripes of modulating width. Due to the fact that as $r_{2}$ increases, the spots become more robust, the influence of the source becomes more localized at the vicinity of the boundaries, and the spots reappear in the center.

We now consider the influence of domain shape on these patterns. In Fig. 5 we show the patterns obtained on a 'fish-like' domain for parameters in the stripe 
(a)

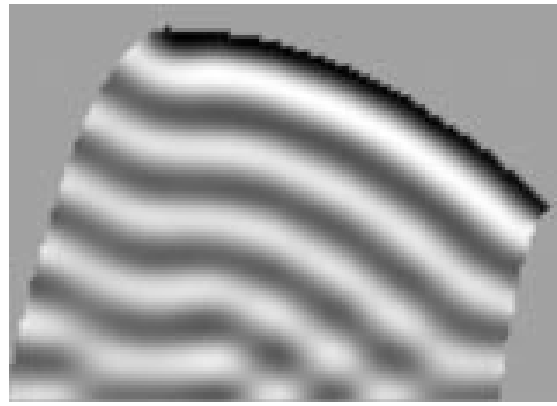

(b)

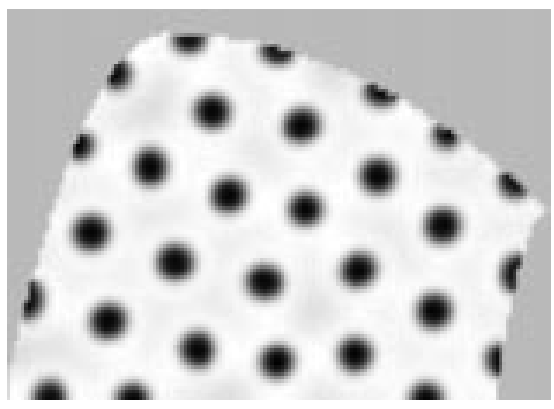

(c)

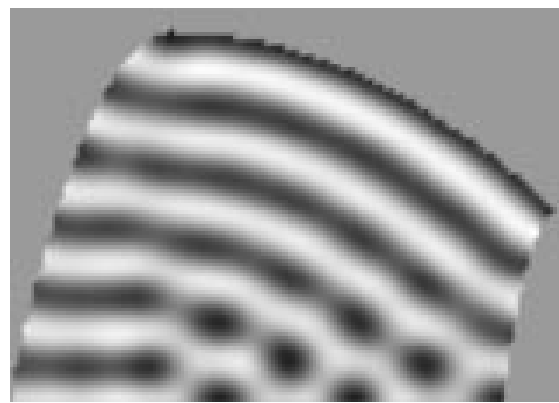

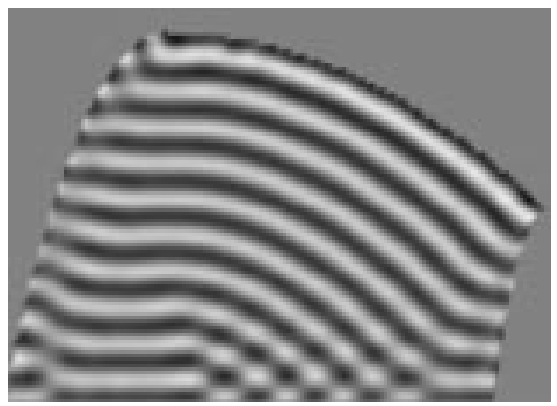
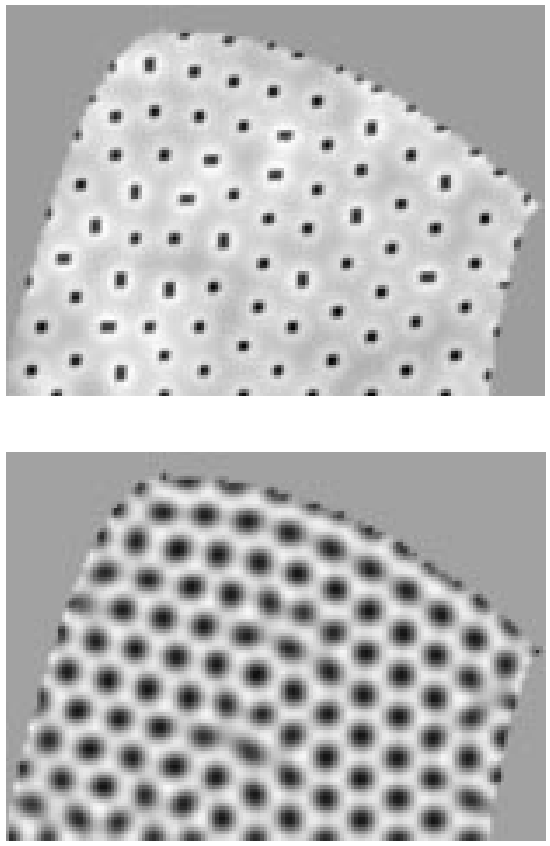

Figure 5. Patterns obtained on a 'fish-like' domain and with a $\operatorname{sink} h=-0.005$ of $u$ at the top curved surface: (a) Parameters as in Fig. 2(a); (b) parameters corresponding to Fig. 2(e); (c) parameters taken from Fig. 3 .

regime, when a source term in added either in the horizontal or the vertical borders. Observe that the stripes align along the source edges. These patterns are very similar to the ones published in Varea et al. (1997), but the model is different. Hence, one can conclude that the important factor here is the presence of the source, and not the peculiarities of the model used.

When the source (or sink) value is very high, the stripes with different orientation emanating from the upper and lower borders collide and a sharp region of transition is observed. The change in spot patterns is similar to that illustrated in Fig. 4. However, when one chooses a regime in which spots and stripes compete, as in Fig. 3, two things happen: first, one stabilizes stripes in the region near the source; second, the pattern with stripes and spots becomes stable. This is shown in the 
bottom panel of Fig. 5. A similar pattern has been obtained by modulating $r_{2}$ with a step function.

\section{INTERACTING SYSTEMS}

In this section we investigate interacting Turing systems. Specifically, we consider a second Turing system in chemicals $\left(u^{\prime}, v^{\prime}\right)$ that modulates the kinetic terms in the $(u, v)$ system to give the model:

$$
\begin{aligned}
& \frac{\partial u}{\partial t}=D \delta \nabla^{2} u+\alpha u\left(1-r_{1} v^{2}\right)+v\left(1-r_{2} u\right)+q_{1} u^{\prime}+q_{2} u^{\prime} v+q_{3} u^{\prime} v^{2} \\
& \frac{\partial v}{\partial t}=\delta \nabla^{2} v+\beta v\left(1+\frac{\alpha r_{1}}{\beta} u v\right)+u\left(\gamma+r_{2} v\right)-q_{2} v^{\prime} u-q_{3} v^{2} u^{\prime} \\
& \frac{\partial u^{\prime}}{\partial t}=D^{\prime} \delta^{\prime} \nabla^{2} u^{\prime}+\alpha^{\prime} u^{\prime}\left(1-r_{1} v^{\prime 2}\right)+v^{\prime}\left(1-r_{2} u^{\prime}\right) \\
& \frac{\partial v^{\prime}}{\partial t}=\delta^{\prime} \nabla^{2} v^{\prime}+\beta^{\prime} v^{\prime}\left(1+\frac{\alpha^{\prime} r_{1}}{\beta^{\prime}} u^{\prime} v^{\prime}\right)+u^{\prime}\left(\gamma^{\prime}+r_{2} v^{\prime}\right) .
\end{aligned}
$$

This allows us to investigate the changes in pattern properties that occur due to the interaction between the two systems. It also enables us to choose two identical systems, each in a different patterning regime, so that we can study, for example, the interaction of patterns with different, or even incommensurate wavelengths; competition between spots and stripes; and to see if new patterns can be obtained, for example, fringes of various widths, spots of varying diameter, etc.

The linear analysis is not affected by $q_{2}$ or $q_{3}$, but when $q_{1}$ is present, the eigenvalues of the linearized system are the same as those shown in Fig. 1. However, the eigenvectors are mixed up by the coupling, in the sense that they contain non-zero components of both primed and unprimed variables. The mode of small wave vector is not greatly affected, but the one with longer $k$, or small wavelength is increasingly mixed as $q_{1}$ gets larger, up to the point that for $q_{1} \approx 0.5$ the $u^{\prime}$ component dominates.

The numerical calculations using this system were all made with parameter values such that the $(u, v)$ system pumps the mode $k=0.42$ and the $\left(u^{\prime}, v^{\prime}\right)$ system pumps the mode $k=0.84$. All the calculations converged to the numerical error of the program $\left(\approx 10^{-7}\right)$.

In Fig. 6 we show results for linear, quadratic and cubic positive interactions. The solution to the non-interacting systems corresponding to these parameters are the ones shown in Fig. 3. Observe that $q_{1}$ and $q_{3}$ alone do not change the spot pattern, but produce defects in the hexagonal lattices, due to the interaction of different wavelengths. The quadratic interaction produces a more striking effect, the spot pattern is now superimposed on a labyrinth pattern of stripes. This type of pattern 
is completely new, and more complex than the former ones. It should be noted that some patterns of the form illustrated in Fig. 6 can be exhibited by a single Turing system [see, for example, Ouyang and Swinney (1991); Dulfiet and Boissonade (1992)].

In our calculations it is always seen that the $\left(u^{\prime}, v^{\prime}\right)$ sub-system converges more rapidly than the $(u, v)$ sub-system. Therefore, the quadratic interaction acts effectively as a modulation of $r_{2}$ in the domain, just as if the first system is reacting on top of a modulated substrate. Figure 7 shows the same calculation but with negative interaction parameters.

As in the case of a single Turing system, we investigated the effect of domain shape on the patterns exhibited by the interacting system by using the same domains as in Fig. 5. The results show that in all cases there was no appreciable change in the patterns. However, when a small source term is added at the top curved boundary, as in Fig. 5, the patterns align themselves to that boundary, and ordered patterns appear. This is shown in Fig. 8 for positive values of the interaction parameters, and in Fig. 9 for negative values.

A linear interaction ( $q_{1}$ non-zero) has very little effect on the pattern, and the spots corresponding to the primed system dominate, and align very close to the sink boundary. However, this case presents the interesting feature that it converges very slowly. On the time scale of embryological development, the transients could become important.

Quadratic interaction ( $q_{2}$ non-zero) is more efficient in aligning the stripe patterns; positive values tend to enhance the stripes, negative values make the spots more apparent. By far the most interesting patterns are obtained with a cubic interaction and a strong sink. Positive values of $q_{3}$ produce a mixed pattern of aligned stripes and spots, and these are complex, resembling 'halo-like' structures. Negative values of $q_{3}$ result in the effects of the sink being felt at a greater distance, resulting in a more pronounced stripy pattern.

\section{Applications and Discussion}

The first application of Turing systems to pigmentation patterns was by Murray (1981). He showed that the patterns exhibited by a system of coupled reactiondiffusion equations with zero flux boundary conditions were similar to those observed on many mammalian coats. He considered the steady-state problem on different sizes of domain and investigated how the solutions varied with scale. For most coat markings, growth simply results in a qualitative change in pattern. However, in certain fish species, dramatic quantitative changes in patterns can occur as the result of growth. This was first modeled by Kondo and Asai (1995), who showed that Turing patterns evolving on a growing domain gave rise to a spatiotemporal sequence of patterns that was consistent with those observed on certain fish species. 
$u$
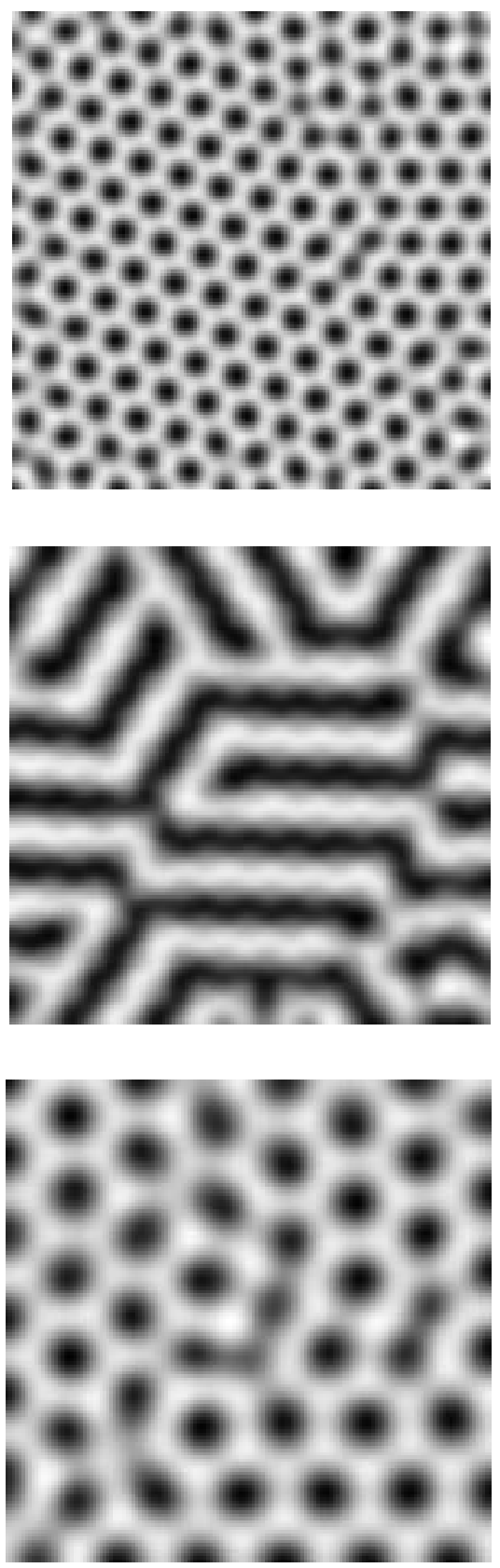

(a)

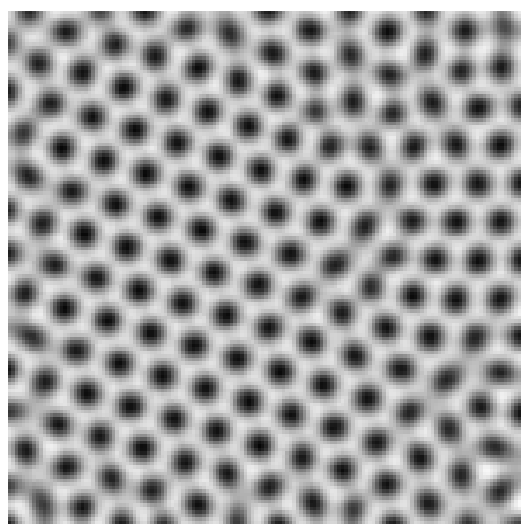

(b)

c)

(e)

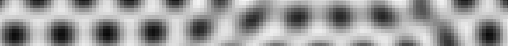

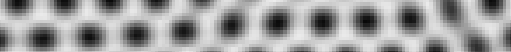

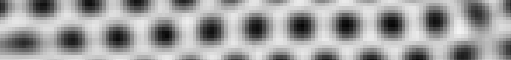

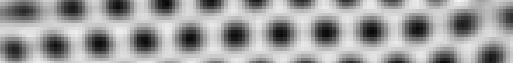

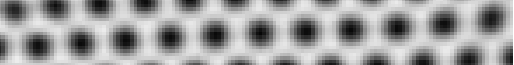

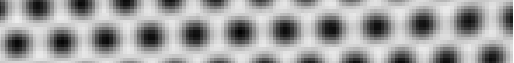

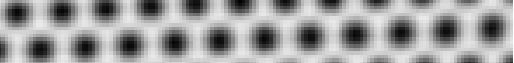

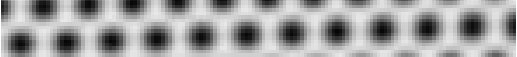

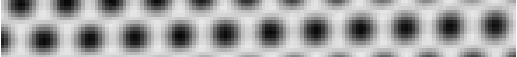

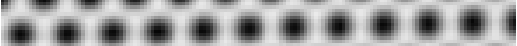

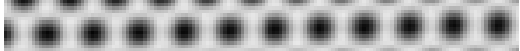

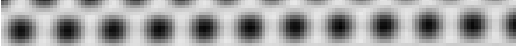
fee e e e e e e e

Figure 6. Patterns obtained with the coupled system equation (3). The parameter sets for $u$ and $u^{\prime}$ are those in Fig. 2(a) and (b), respectively. The top row has only linear coupling ( $q_{1}$ non-zero), the middle row has only quadratic coupling ( $q_{2}$ non-zero), and the bottom row has only cubic coupling ( $q_{3}$ non-zero). In all cases, the non-zero value of the coupling is taken to be 0.55 . Observe that the $u$ pattern is completely destroyed with linear coupling and is identical to $u^{\prime}$. Quadratic coupling favors a labyrinth pattern, while cubic coupling produces only spots. 

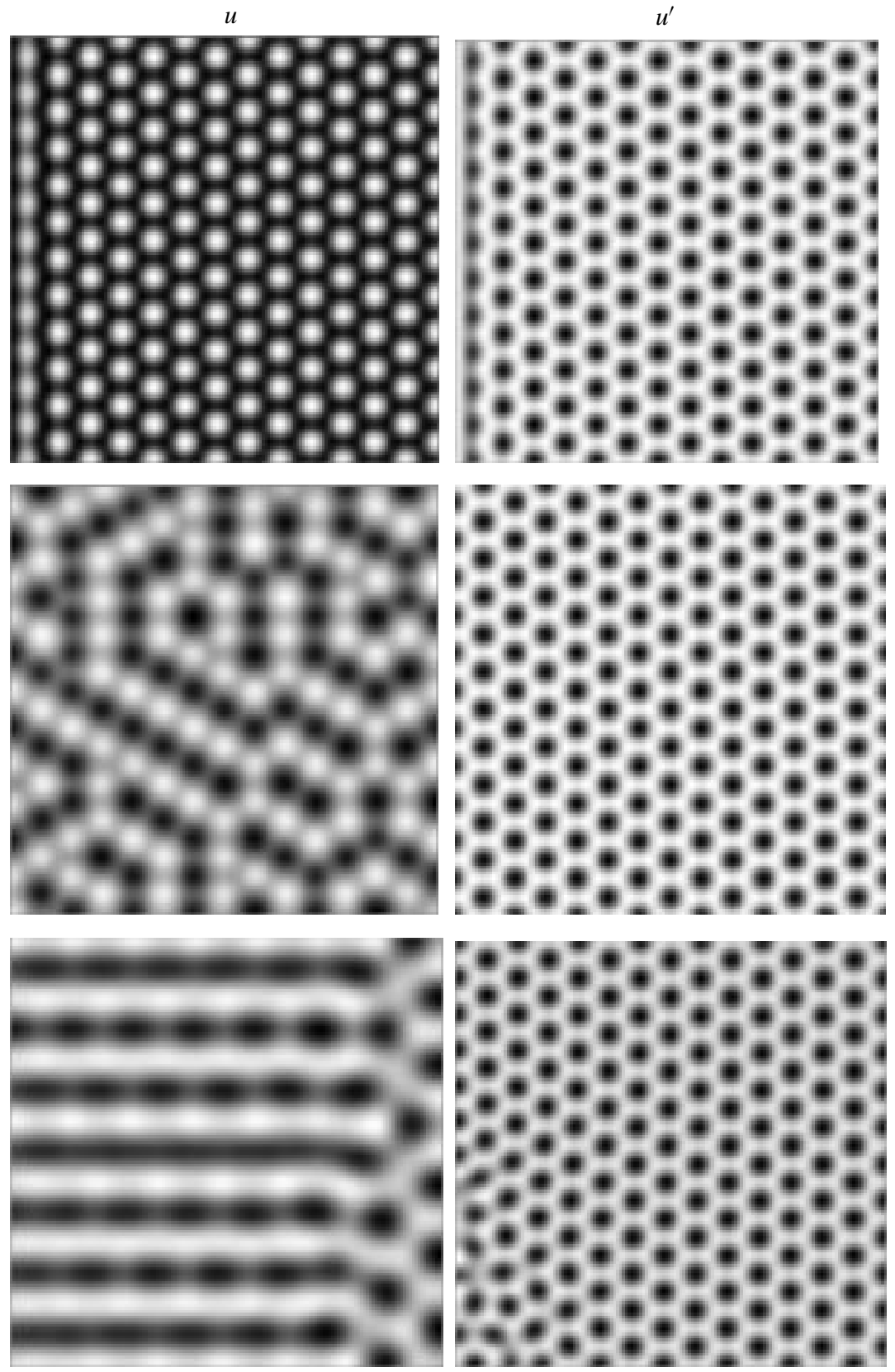

Figure 7. Same as Fig. 6, but with negative couplings $q_{i}=-0.55$. Observe that now the linear term produces the negative image of $u^{\prime}$ in $u$. The other couplings produce a superposition of stripes and spots in the $u$ pattern. The quadratic coupling now favors spots, and the cubic coupling produces ordered spots.

The above studies considered the boundary as an impermeable barrier to the chemical morphogens. Nijhout (1990) considered the effect of boundary and internal 
$u$
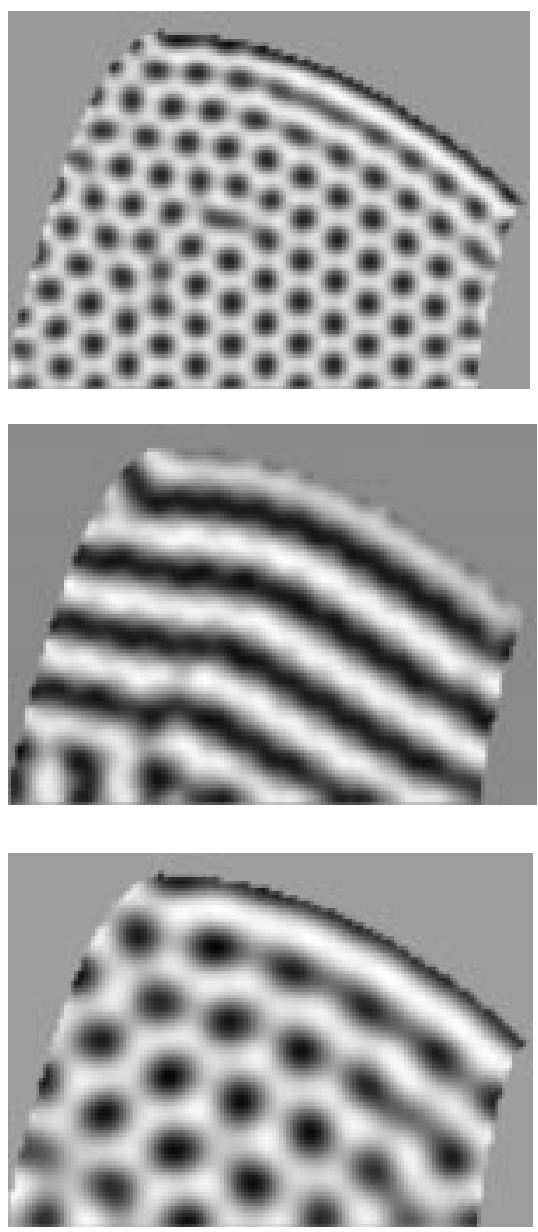

$u \prime$
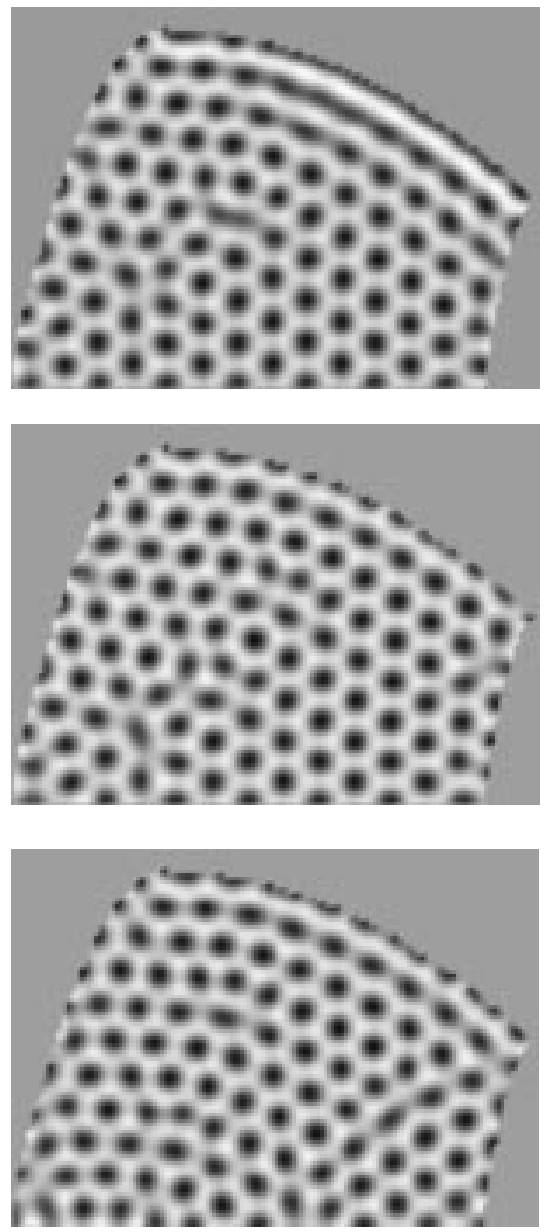

Figure 8. Simulations of the interacting system on a 'fish' shaped domain with parameter values as in Fig. 6, and with a sink term $h$ for $u$ at the top boundary. For linear coupling $h=-0.05$, for quadratic coupling $h=-0.005$ and for cubic coupling $h=-0.1$. Observe that the linear coupling is very insensitive to the sink, while the quadratic coupling forms oriented stripes parallel to the sink. Cubic coupling produces aligned stripes near the sink, but complex spots far away from the sink boundary.

source terms with respect to the application of reaction-diffusion theory to butterfly wing patterns. More recently, Varea et al. (1997) have considered the effect of boundary source terms and geometry on the patterns exhibited by reaction-diffusion systems on growing domains.

In this paper, we have extended this study to consider in more detail the rôle of boundary source/sink terms and also to investigate the patterns exhibited by interacting reaction-diffusion systems. We have shown that these can give rise to a number of patterns that cannot be exhibited by the standard Turing model. For 
$u$
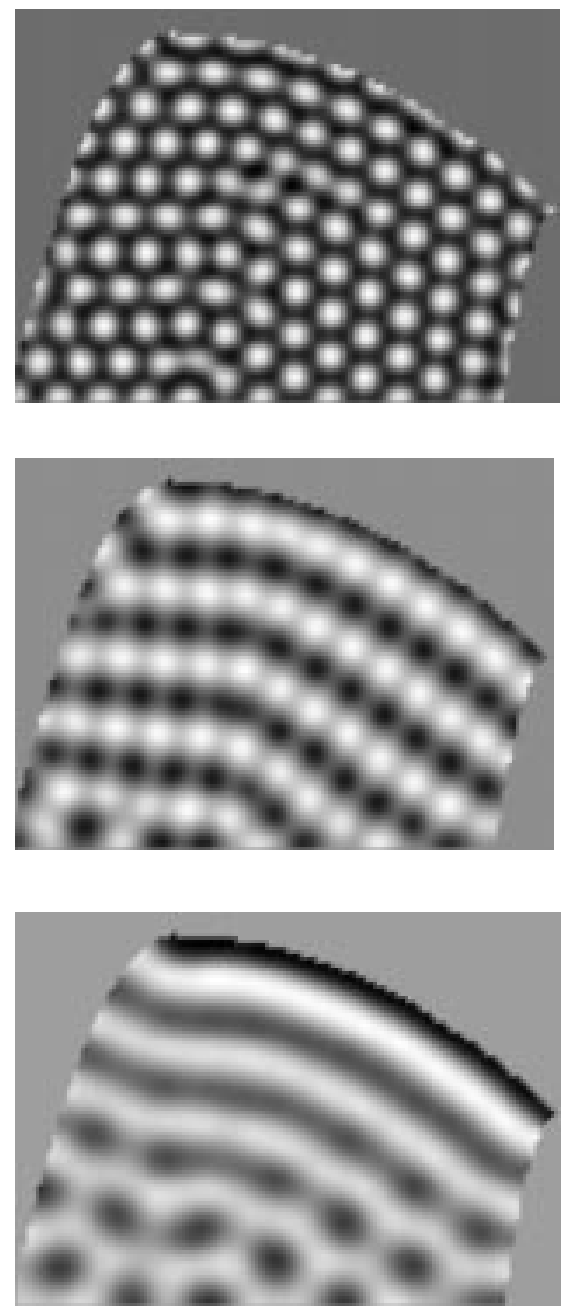

$u^{\prime}$
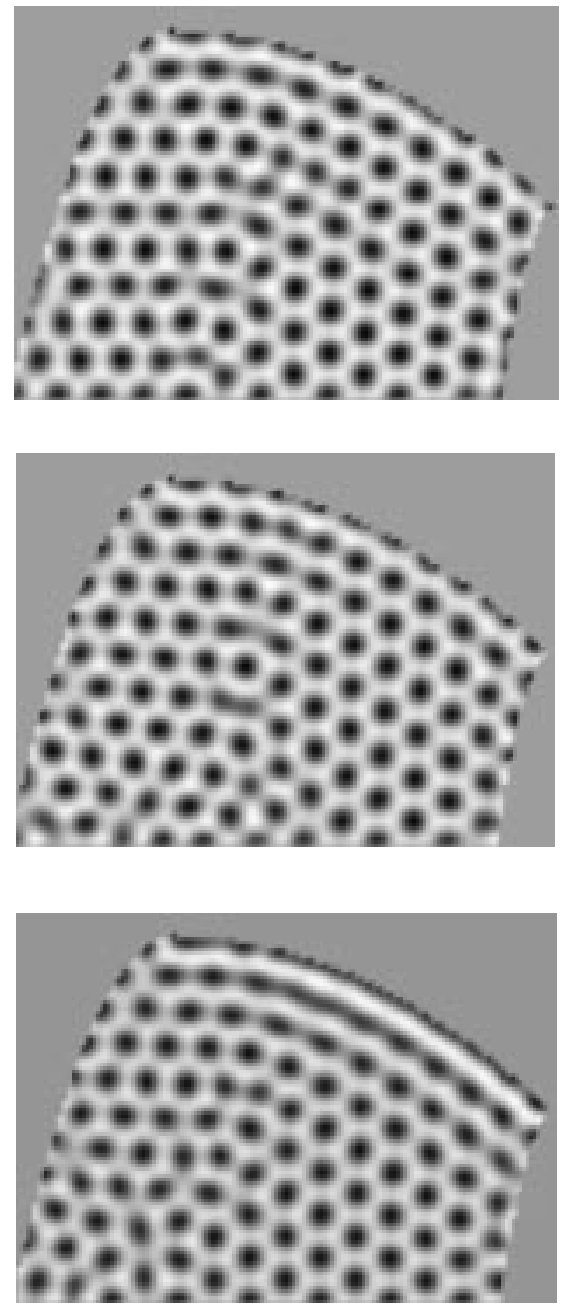

Figure 9. As in Fig. 8, but with negative couplings $q_{i}=-0.55$. Observe that now the linear term produces the negative image of $u^{\prime}$ in $u$. The other couplings produce a superposition of aligned stripes parallel to the sink boundary in the $u$ pattern. The quadratic coupling now favors spots, and the cubic coupling produces a combined pattern of stripes and spots.

example, in some of the simulations, we incorporated spatially-varying parameters into the model and investigated the spatial patterns exhibited by the resulting Turing system. The natural question then arises as to how the spatial pattern in parameters was set up. This can be thought of as the output of a second Turing system with a very short characteristic diffusion time, so that a quasi-steady-state assumption can be used. In Fig. 10, a reaction-diffusion system with spatially-modulated parameters produces rows of spots interspersed with stripes. This extends the ideas of Benson (1994), and the patterns are similar to those observed on the thirteen-lined 


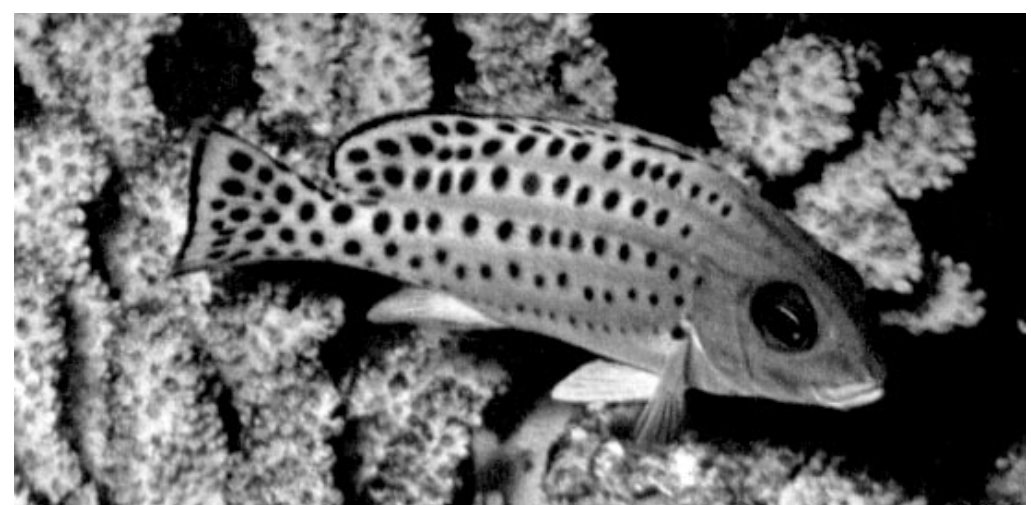

Pomacanthus maculatus

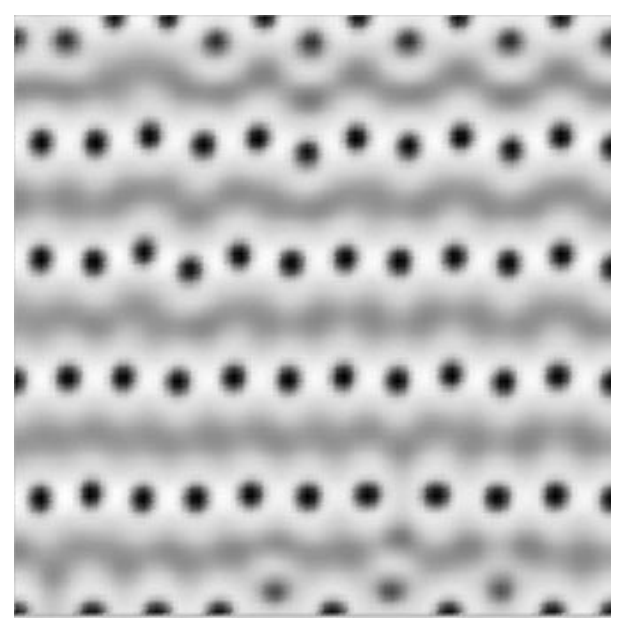

Figure 10. Pattern obtained with equation (2) for parameter values $r_{1}=1.5$ and $r_{2}=\cos \pi y / 10, D=0.182, \alpha=0.5$, and $\beta=-0.578$, which enhance modes $\bar{k}=2 \pi(5 / L, 5 / L)$ and $2 \pi(7 / L, 0)$. This kind of parameter modulation produces an alternate pattern of stripes and spots which cannot be produced by a standard Turing mechanism. Note that this pattern resembles the pigmentation on the fish Pomacanthus maculatus [reproduced from Frank (1973)].

ground squirrel (Bard, 1981; Cocho et al., 1987). These patterns are also similar to those observed on Pomacanthus maculatus. In Fig. 10 we show a pattern obtained with a sinusoidal modulation of $r_{2}$.

We have shown that boundary source/sink terms can also play a crucial rôle in pattern selection. For example, the striped pattern breaking into spots in Fig. 5 closely resembles that observed on the fish Siganus vermiculatus (Fig. 11).

A pattern very similar to the one shown in the second row of Fig. 6 is actually found, with two different wavelengths, in the Hypostomus plecostomus, as exhibited in Fig. 12, and the one found in the first row of Fig. 5 is actually found in the Zebrasoma desjardinii (Fig. 13). 


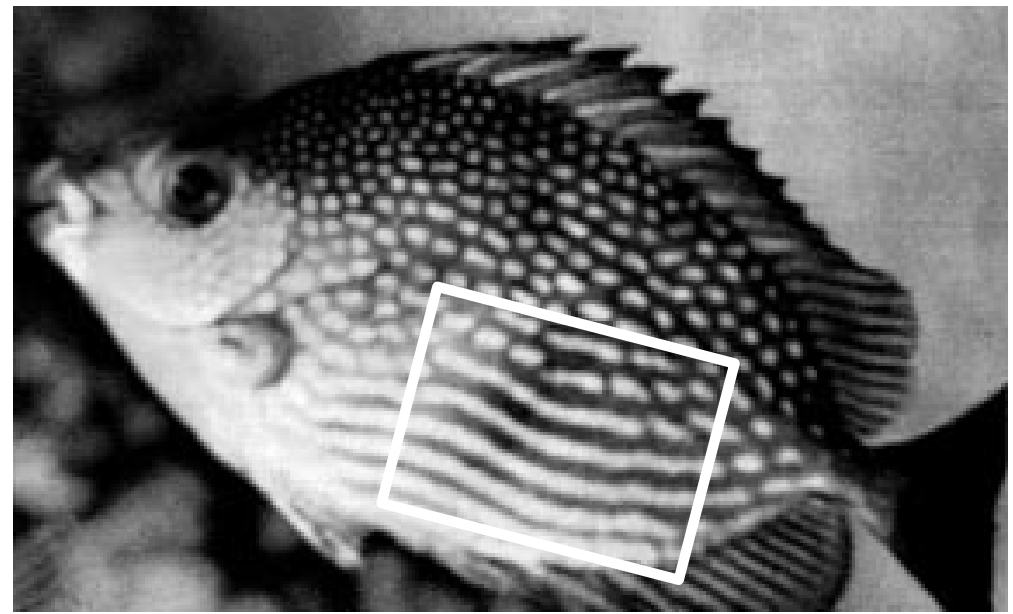

Siganus vermiculatus

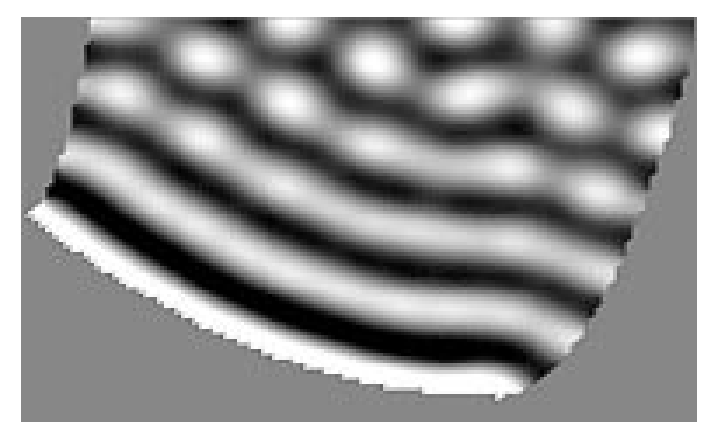

Figure 11. The skin pattern of the Siganus vermiculatus [reproduced from Frank (1973)], showing the transition from stripes to spots that can be simulated with the calculations shown in Fig. 5 when a source is present at one of the boundaries.

An example of a transient pattern is shown in Fig. 14, obtained with a small $q_{1}$ coupling, which converges very slowly, and compared with the hexagons with missing central spots found in the skin of the Coria formosa parrot fish.

A very important aspect of pattern generation is the robustness of pattern. This depends on a number of things, including the boundary conditions and the nonlinear terms. Our results show that in the case where spots compete with stripes, spotted patterns are very robust and appear to arise as long as the coefficient of the quadratic term is non-zero (Fig. 3 illustrates this). A source-type boundary condition can robustly select stripes near to the boundary in the case when there is stripe-spot competition (Figs 4 and 8). For the case where stripes are the only pattern, the boundary sources can influence their orientation (illustrated by Fig. 13). 


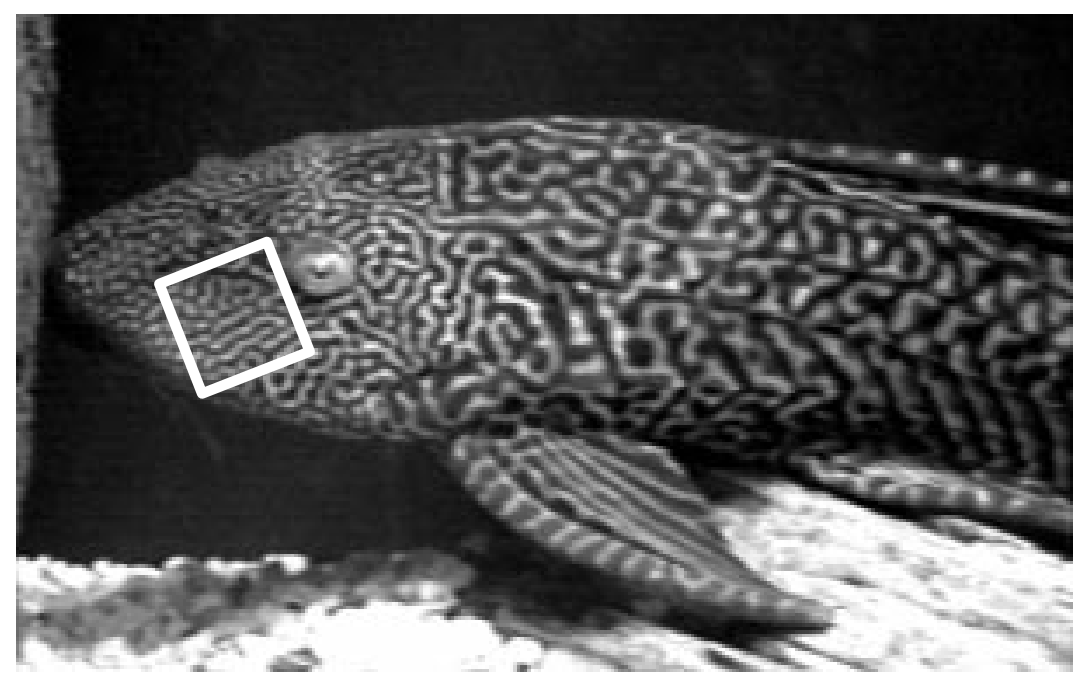

Hypostomus plecostomus

(a)

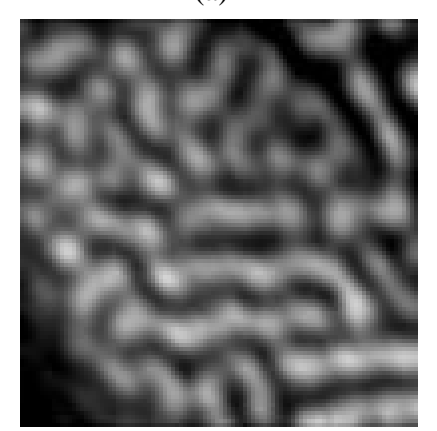

(b)

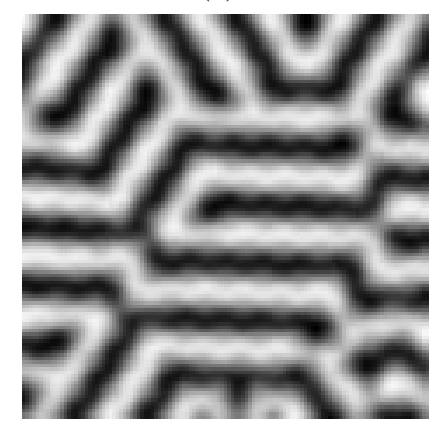

Figure 12. The skin pattern of the Hypostomus plecostomus can be modeled with the set of quadratically coupled Turing equations (see Fig. 6).

\section{Conclusions}

In this paper, we have carried out a detailed numerical investigation of a generic Turing system and have examined the effects of boundary conditions, domain shape, and coupling of Turing systems. We have shown that these modified models can generate patterns not exhibited by the standard Turing model, and have compared them with the pigmentation patterns observed on fish. For example, coupled Turing systems with very different diffusion times can exhibit stripes interspersed with spots (Fig. 10) due to the modulation of the non-linear terms which are crucial in determining whether the pattern evolves to stripes or to spots. Also, the coupled system can exhibit hexagonal arrays of spots with the central spot missing (Fig. 14). We are unaware of such patterns arising from a single Turing system. Coupled Turing systems are able to produce modulated stripes [Figs 6(c) and 7]. Furthermore, 


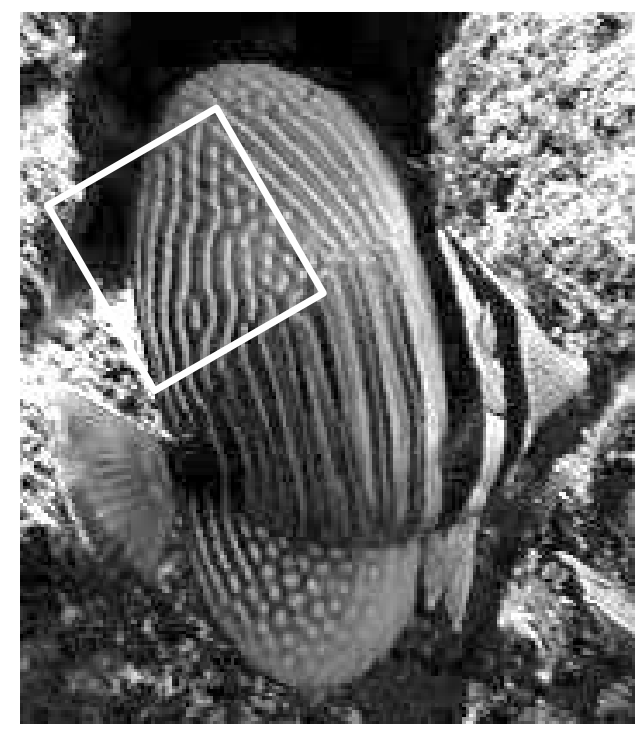

Zebrasoma desjardinii

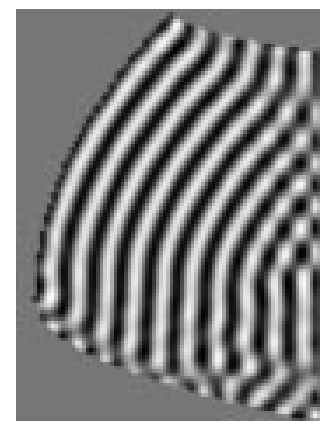

Figure 13. Complex patterns of stripes and spots can be obtained with our model with sources, and are found in real fish, as the complicated encounter of two sets of stripes with different orientation in the skin of the surgeon fish Zebrasoma desjardinii. The comparison is made with the theoretical pattern of Fig. 5.

if the parametrically enhanced wavelengths are incommensurate, one can obtain non-periodic patterns.

Presently, there is no firm biological evidence that pigmentation patterning in fish arises due to a chemical pre-pattern. It has been suggested [see Twitty and Niu (1954) and references therein] that pigmentation patterns may arise due to cell movement in response to diffusible factors secreted by cells. This type of pattern formation mechanism is presently under investigation (Painter et al., 1999). The results of our present paper show that a comparatively simple model can produce a suprisingly diverse range of complex patterns. Our model simulations show that boundary source terms, domain size, and domain shape can play crucial roles in pattern selection. These ideas could be tested experimentally by placing barriers near the hypothesized sources to determine if different patterns evolve. Do- 


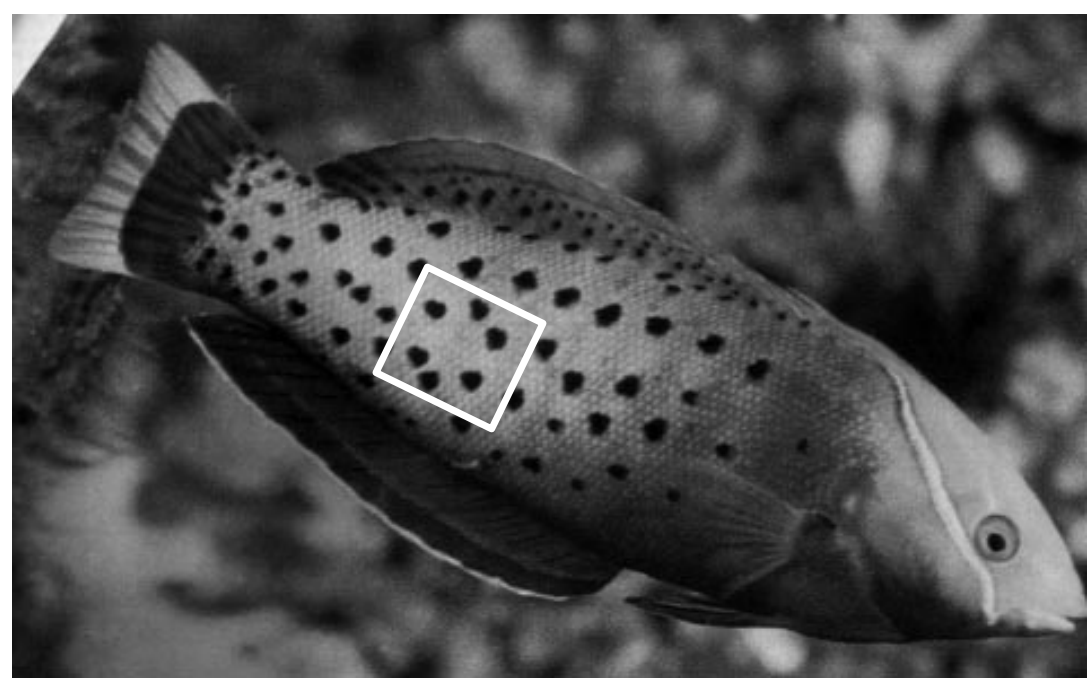

Coris formosa

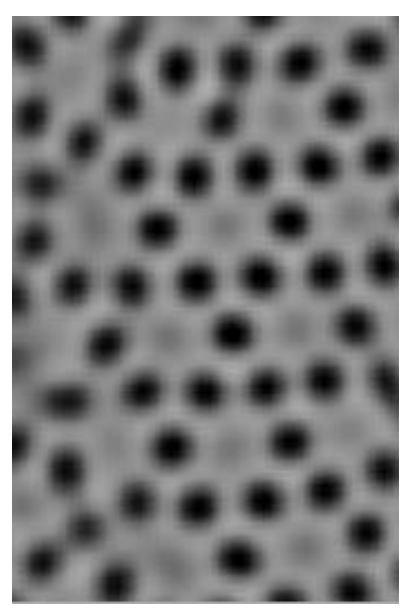

Figure 14. Transient pattern obtained after 20000 iterations, using the same parameters as in Fig. 11, except that $r_{1}=3.5$ and $r_{2}=0.47$. Only linear coupling $q_{1}=-0.2$ is present. This pattern converges very slowly and the transient pattern is very complicated and disordered. This pattern presents hexagons with missing centers as the ones found on the skin of the fish Coria formosa [reproduced from Frank (1973)].

main size and shape could be altered by varying feeding. Although we explicitly studied a reaction-diffusion model, we believe that our results will hold for any short-range activation, long-range inhibition model. We conclude that such a mechanism may play a rôle in pigmentation patterning in fish. We are currently investigating more fully the regions in parameter space where different patterns occurrs. 


\section{ACKNOWLEDGEMENTS}

We acknowledge financial support from UNAM through projects No. IN-104296 and IN-107296, and from CONACyT through project 25237-E. Part of this work was carried out while PKM was visiting UNAM under the scheme funded by the Royal Society (London) and Mexican Academy of Sciences. We thank DGSCA (UNAM) for allowing use of their super-computing facilities.

\section{REFERENCES}

Bard, J. B. L. (1981). A model for generating aspects of zebra and other mammalian coat patterns. J. Theor. Biol. 93, 363-385.

Benson, D. L. (1994). Reaction-diffusion models with spatially inhomogeneous diffusion coefficients, Ph.D. thesis, Oxford University, Oxford.

Bunow, B., J. P. Kernevez, G. Joly and D. Thomas (1981). Pattern formation by reactiondiffusion instabilities: application to morphogenesis in Drosophila. J. Theor. Biol. 84, 629-649.

Cocho, G., R. Pérez-Pascual and J. L. Rius (1987). Discrete systems, cell-cell interactions and color pattern of animals. I. Conflicting dynamics and pattern formation. J. Theor. Biol. 125, 419-435.

Cruywagen, G. C., P. K. Maini and J. D. Murray (1992). Sequential pattern formation in a model for skin morphogenesis. IMA J. Math. Appl. Med. Biol. 9, 227-248.

Cruywagen, G. C., P. K. Maini and J. D. Murray (1997). Biological pattern formation on two-dimensional domains: A non-linear bifurcation analysis. SIAM J. Appl. Math. 57, 1485-1509.

Cruywagen, G. C. and J. D. Murray (1992). On a tissue interaction model for skin pattern formation. J. Nonlinear Sci. 2, 217-240.

Dillon, R., P. K. Maini and H. G. Othmer (1994). Pattern formation in generalised Turing systems: I. Steady-state patterns in systems with mixed boundary conditions. J. Math. Biol. 32, 345-393.

Dufiet, V. and J. Boissonade (1992). Numerical studies of Turing patterns selection in a two-dimensional system. Physica A188, 158-171.

Ermentrout, B. (1991). Stripes or spots? Non-linear effects in bifurcation of reactiondiffusion equations on the square. Proc. R. Soc. Lond. A434, 413-417.

Frank, S. (1973). Encyclopédie illustrée des Poissons, Paris: Gründ.

Höfer, T. and P. K. Maini (1997). Streaming instability in dictyostelium revisited. Phys. Rev., E56, 2074-2080.

Kondo, S. and R. Asai (1995). A reaction-diffusion wave on the skin of the marine angelfish Pomacanthus. Nature 376, 765-768.

Maini, P. K., K. J. Painter and H. N. P. Chau (1997) Spatial pattern formation in chemical and biological systems. Faraday Trans. 93, 3601-3610.

Murray, J. D. (1981). A pre-pattern formation mechanism for animal coat markings. J. Theor. Biol. 88, 161-199.

Murray, J. D. (1993). Mathematical Biology, Berlin: Springer Verlag. 
Murray, J. D., G. C. Cruywagen and P. K. Maini (1994). Pattern formation in tissue interaction models, in Frontiers in Mathematical Biology, S. A. Levin (Ed.), Lecture Notes in Biomathematics 100, 104-116.

Nagorcka, B. N., V. S. Manoranjan and J.D. Murray (1987). Complex spatial patterns from tissue interactions_-an illustrative model, J. Theor. Biol. 128, 359-374.

Nagorcka, B. N. and J. R. Mooney (1992). From stripes to spots: prepatterns which can be produced in the skin by a reaction-diffusion system. IMA J. Math. Appl. Med. Biol. 9, 249-267.

Nijhout, H. F. (1990). A comprehensive model for colour pattern formation in butterflies. Proc. R. Soc. Lond. B239, 81-113.

Ouyang, Q. and H. L. Swinney (1991). Transition from a uniform state to hexagonal and striped Turing patterns. Nature 352, 610-612.

Painter, K. J., H. G. Othmer and P. K. Maini (1999). Stripe formation in juvenile Pomacanthus explained by a generalized Turing mechanism with chemotaxis (submitted).

Shaw, L. J. and J. D. Murray (1990). Analysis of a model for complex skin patterns. SIAM J. Appl. Math. 50, 628-648.

Turing, A. M. (1952). The chemical basis of morphogenesis, Phil. Trans. R. Soc. Lond. B237, 37-72.

Twitty, V. C. and M. C. Niu (1954). The motivation of cell migration, studied by isolation of embryonic pigment cells singly and in small groups in vitro. J. Exp. Zoo. 108, 405-437.

Varea, C., J. L. Aragón and R. A. Barrio (1997). Confined Turing patterns in growing systems Phys. Rev. E56, 1250-1253. 